\title{
Comportamento fotofísico do antraceno em sistemas micro- heterogêneos argila-surfactantes-íons metálicos
}

\author{
R. Magri, F.L.Fertonani, I.A. Pastre* \\ Instituto de Química, Unesp, Campus de Araraquara, CEP 14801-970 - Araraquara - SP - Brasil \\ refez@ig.com.br;fertonan@iq.unesp.br; *pastre@ibilce.unesp.br
}

\begin{abstract}
Resumo: Argilas constituem uma classe de complexos micro-heterogêneos e podem ser utilizados como substrato para adsorção. O seu comportamento de sorção em fase sólida intensificada pela presença de surfactantes, argilas organofílicas, é um importante fenômeno explorado pela tecnologia ambiental para a remoção de compostos orgânicos policíclicos (hidrocarbonetos aromáticos policíclicos, HPA) da água, introduzidos no ambiente por fontes antropogênicas. Este trabalho tem por objetivo estudar o comportamento fotofísico do antraceno, como modelo de HPA, em sistemas micro-heterogêneos argila-surfactantes-íons metálicos (M(II)= Cd(II), $\mathrm{Cu}(\mathrm{II}), \mathrm{Hg}(\mathrm{II}), \mathrm{Ni}(\mathrm{II})$ e $\mathrm{Pb}(\mathrm{II})$; surfactantes: CTACl; SDS; TR-X100). Os estudos foram conduzidos pelo monitoramento na mudança das propriedades de fluorescência estática e na supressão da emissão do antraceno utilizado como sonda fluorescente. Como supressores foram utilizados os cátions metálicos: $\mathrm{Cd}(\mathrm{II}), \mathrm{Cu}(\mathrm{II}), \mathrm{Hg}(\mathrm{II}), \mathrm{Ni}(\mathrm{II})$ e $\mathrm{Pb}(\mathrm{II})$. O perfil do espectro de fluorescência e os resultados dos ensaios de supressão da fluorescência da sonda permitiram inferir na localização do sítio de solubilização do antraceno nos sistemas micro-heterogêneos estudados e na conseqüente organização dos mesmos.
\end{abstract}

Palavras-chave: antraceno; argila; supressão; fluorescência; íons metálicos.

\section{Introdução}

O estudo fotoquímico e fotofísico de moléculas sondam em sistemas micro-heterogêneos inorgânicos são de grande interesse, de modo que moléculas tais como tris(2,2'-bipiridina) rutênio(II), pireno e derivados de pireno, antraceno e derivados, 8-hidroxiquinolina e corantes são muito utilizadas como sondas fluorescentes para a obtenção de propriedades físico-químicas dos diferentes microambientes formados em matrizes de sílica [1;2], argilas $[3 ; 4 ; 14 ; 15]$ e óxidos $[5 ; 6]$, dentre outras.

Argilominerais são atrativos para aplicações desta natureza devido a sua grande área de superfície, capacidade de inchamento, de troca iônica, sua abundante ocorrência na natureza, e seu comportamento catalítico. O mais importante mecanismo de adsorção na superfície da argila é o de troca de íons por moléculas carregadas positivamente. Os estudos dos processos de adsorção envolvem: 1) a velocidade de adsorção, 2) a distribuição de cátions adsorvidos sobre a superfície das partículas de argila, especialmente à baixa cobertura, 3 ) a distribuição de moléculas adsorvidas nas diferentes partículas de argila e nos espaços interlamelares [13].

A coadsorção de moléculas como os surfactantes na superfície da argila é uma forma de solubilizar moléculas apolares, o que pode ser evidenciado nos experimentos fotoquímicos, bem como interferir na estabilidade coloidal dos sistemas aquosos, na interação partícula-partícula e na formação de sedimentos [7]. Assim, nanocom- 
pósitos formados por argila-surfactante, são materiais que apresentam propriedades de interesse científico e tecnológico $[8 ; 9 ; 10]$.

Existem uns conjuntos significativos de técnicas físico-químicas empregadas para o estudo de superfícies e superfícies modificadas, tais como: análise termogravimétrica, espectroscopia na região do infravermelho, ressonância magnética, difração de raios $\mathrm{X}$, dentre outras. A observação de processos que ocorrem nos estados excitados de moléculas adsorvidas na interface argilomineral-moléculas orgânicas tem sido investigada empregando-se a técnica de fluorescência na região do Uv-visível.

As moléculas dos compostos utilizados como sondas fotofísicas e fotoquímicas são aquelas que sofrem variações nas suas propriedades de emissão (deslocamentos espectrais ou variações temporais) dependendo do meio reacional em que se encontram. Estas variações se devem a reações no estado excitado, alterações na interação solutosolvente, as quais podem afetar a população relativa em vários níveis vibracionais do estado excitado, sendo manifestada por aparecimento de uma nova banda, variação na intensidade das bandas e deslocamentos espectrais para o azul ou o vermelho freqüentemente acompanhados por mudanças no rendimento quântico de fluorescência $\left(\phi_{\mathrm{F}}\right)$, que é a relação de fótons emitidos pelos absorvidos $[11 ; 13 ; 14]$.

As técnicas de investigação usando sondas fluorescentes ou fosforescentes são importantes, por serem sensíveis ao meio e empregadas em baixa concentração, não perturbando o sistema em estudo, sendo muito utilizadas na determinação da concentração micelar crítica, número de agregação em sistemas micelares, distribuição de solutos, características dos microambientes nos sistemas micro-heterogêneos e efeito de agregados micelares e polieletrólitos nas cinéticas de reações.[8,12-13]

Sistemas desta natureza são atrativos porque a fluorescência é um evento que ocorre em uma escala de tempo muito menor do que os processos de cinética micelar. Assim, um processo de supressão ocorre mantendo a micela intacta, não ocorrendo à saída da sonda, nem destruição da micela durante o processo. Antraceno, naftaleno e pireno, entre outros, são hidrocarbonetos aromá- ticos que têm sido muito utilizados como sondas fotoquímicas para investigar a superfície de argilas. A adsorção destes compostos nas superfícies das matrizes modifica significativamente suas propriedades fotoquímicas e fotofísicas.

Desta forma, toda a química dos colóides, atualmente, está associada à fotoquímica porque a técnica de investigação empregando-se de sondas fluorescentes ou fosforescentes permite a determinação das propriedades químicas e físicas dos sistemas micro-heterogêneos.

Este trabalho tem por objetivo estudar o comportamento fotofísico do antraceno, como modelo de HPA, em sistemas micro-heterogêneos argila-surfactantes-íons metálicos $(\mathrm{M}(\mathrm{II})=\mathrm{Cd}(\mathrm{II})$, $\mathrm{Cu}(\mathrm{II}), \mathrm{Hg}(\mathrm{II}), \mathrm{Ni}(\mathrm{II})$ e $\mathrm{Pb}(\mathrm{II})$; surfactantes: CTACl; SDS; TR-X100), de modo a se contribuir para o entendimento do processo de acomodação das moléculas de antraceno e de surfactantes na superfície dos colóides de natureza inorgânica.

\section{Material e Métodos}

$\mathrm{O}$ antraceno, previamente purificado por sublimação [11], foi utilizado como sonda por apresentar um alto rendimento quântico de fluorescência e variação espectral em função da polaridade do meio. Tais propriedades o tornam um potente sonda na investigação de sistemas micro-heterogêneos [11]. Assim, os resultados das medidas da variação espectral e de supressão de fluorescência foram utilizados para definir os locais de solubilização da sonda e as forças eletrostáticas e ou hidrofóbicas que atuam na interação surfactante-argila. Os surfactantes utilizados nos estudos apresentavam carga idêntica (aniônicos) e contrária (catiônicos) à da argila; foi incluído surfactante não iônico para comparação. Na obtenção dos dados procurou-se estabelecer uma análise comparativa do comportamento espectral (intensificação ou deslocamento de bandas de fluorescência) e da eficiência da supressão da fluorescência da sonda pela presença de cátions metálicos, em água e em suspensão aquosa da argila. As medidas espectrofluorimétricas em sistemas aquosos e nos sistemas micro-heterogêneos foram obtidas no tempo zero da adição da sonda. O tempo zero 
é definido operacionalmente como: adição da sonda, homogeneização e posterior leitura.

As medidas espectrofluorimétrica foram realizadas em um espectrofluorímetro Hitachi, modelo F 4500, empregando-se $\lambda_{\text {excitação }}=260 \mathrm{~nm}$, cubeta de quartzo de $1,00 \mathrm{~cm}$ de caminho óptico e leitura da luz de emissão a $90^{\circ}$ em relação à excitação. As leituras foram realizadas a temperatura de $(25 \pm 2){ }^{\circ} \mathrm{C}$.

\section{Preparação dos sistemas antraceno - água, an- traceno-surfactante, antraceno-argila e antrace- no-surfactante-argila}

Foram preparadas soluções de: a) antraceno $\left(1 \times 10^{-6} \leq \mathrm{C}_{\text {sonda }} \leq 1 \times 10^{-4}\right) \mathrm{mol} \mathrm{L}^{-1}$ diretamente na cubeta da amostra, pela adição de volume adequado de solução estoque de antraceno $\left(3 \times 10^{-3} \mathrm{~mol} \mathrm{~L}^{-1}\right)$ para um volume final de 3,00 $\mathrm{mL}$; b) mistura antraceno-surfactante a partir da adição de solução estoques de surfactante $\left(0,10 \mathrm{~mol} \mathrm{~L}^{-1}\right)$ à solução de antraceno $\left(\mathrm{C}_{\text {final }}=\right.$ $3,0 \times 10^{-5} \mathrm{~mol} \mathrm{~L}^{-1} ; \mathrm{v}_{\text {final }}=3,00 \mathrm{~mL}$ ) de modo a se obter valores de concentrações de surfactantes abaixo e acima da CMC (valores de CMC: SDS - 8,0 X 10-3; CTACl - 1,4 x 10 $0^{-3}$; TRITON X-100 $\left.2,6 \times 10^{-4} ; \mathrm{CP}_{\mathrm{y}} \mathrm{Cl}-8,0 \times 10^{-4} \mathrm{~mol} \mathrm{~L}^{-1}\right)$ [12]; c) antraceno $\left(1 \times 10^{-6} \leq \mathrm{C}_{\text {sonda }} \leq 1 \times 10^{-4}\right) \mathrm{mol} \mathrm{L}^{-1}$ diretamente na cubeta da amostra, conforme descrito em a), porém, contendo suspensão de argila $\left(0,11 \mathrm{~g} \mathrm{~L}^{-1}\right)$; d) mistura antraceno-surfactante, conforme descrito em b),porém, adicionados a suspensão de $\operatorname{argila}\left(0,11 \mathrm{~g} \mathrm{~L}^{-1}\right)$.

Estudos de supressão de fluorescência pela presença de íons $\mathbf{M}$ (II) $(\mathbf{M}=\mathrm{Ni}(\mathrm{II}), \mathrm{Cu}(\mathrm{II})$, $\mathrm{Pb}(\mathrm{II}), \mathrm{Cd}(\mathrm{II})$ e $\mathrm{Hg}(\mathrm{II}))$

Os experimentos de supressão foram obtidos pela adição de solução do íon $\left(1,7 \times 10^{-4} \leq\right.$ $\mathrm{M}(\mathrm{II}) \leq 1,9 \times 10^{-2}$ ) $\mathrm{mol} \mathrm{L}^{-1}$ as soluções preparadas como descrito no item anterior $(\mathbf{a})-\mathbf{d})$ ).

\section{Reagentes}

Foi empregada como sonda fluorescente o antraceno (Aldrich). Os agentes surfactantes Dodecil sulfato de sódio ( SDS), aniônico (Mallinckrodt); cloreto de cetil-trimetil-amônio, catiônico (CTACl) (Aldrich); Cloreto de cetil piridinio (CPyCl), catiônico (Aldrich); Triton X - 100 (T $\mathrm{X}-100)$, não iônico (Reagen), foram empregados sem purificação prévia. A argila Montmorilonita Natural (SWY-1) foi obtida da Source Clays, Clay Mineral Society, University of Missouri, Columbia, M.O.. Os sais de $\mathrm{NiCl}_{2}, \mathrm{CuSO}_{4}, \mathrm{~Pb}\left(\mathrm{NO}_{3}\right)_{2}$, $\mathrm{CdCl}_{2}, \mathrm{HgCl}_{2}$, foram empregados para o preparo das soluções dos supressores sem prévia purificação (Merch).

\section{Resultados e discussão}

\section{Comportamento do sistema antraceno-água}

Os estudos da emissão de fluorescência do antraceno, em fase aquosa, foram realizados em função do incremento da concentração e estão apresentados na Figura 1. Os espectros apresentados na Figura 1a revelam que as intensidades dos componentes vibrônicos das bandas nos comprimentos de onda acima de $400 \mathrm{~nm}$ aumentam com o aumento da concentração, enquanto que para a banda (I) em $380 \mathrm{~nm}$ o decréscimo da intensidade de fluorescência se deve ao efeito de reabsorção da emissão. A intensidade relativa de fluorescência obtida do espectro normalizado, Figura 1b, sugere que o decréscimo acentuado da emissão da espécie monomérica em $380 \mathrm{~nm}$ (I) e o incremento da emissão do agregado em $445 \mathrm{~nm}$ (IV) estão atrelados ao aumento de concentração local e são indicativos da presença de excímeros estáticos, espécies pré-associadas no estado fundamental em solução aquosa. 


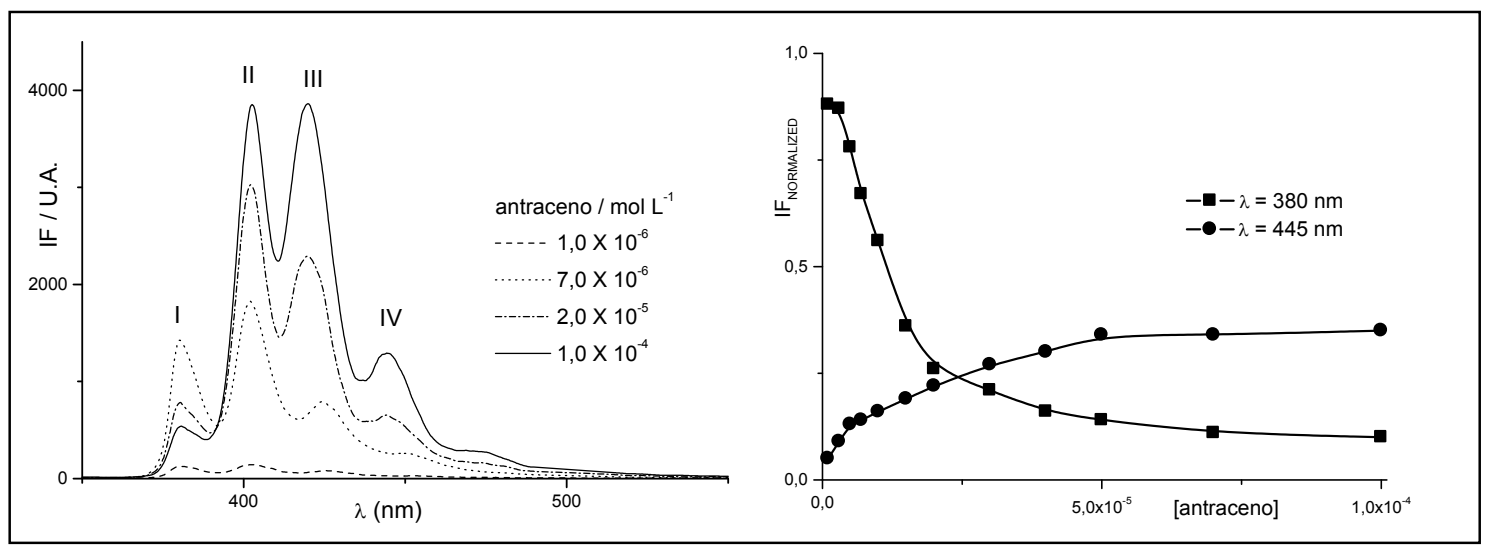

Figura 1. a) Espectro de fluorescência do antraceno em solução aquosa; b) intensidade de fluorescência normalizada em função do aumento da concentração do antraceno em solução aquosa, $\lambda_{\text {exc }}=260 \mathrm{~nm}$.

\section{Comportamento do sistema antraceno-surfactantes}

Os espectros de fluorescência do antraceno em função do aumento da concentração dos surfactantes estão apresentados na Figura 2 a-d. Observa-se a partir da figura que a intensidade de emissão aumenta com o aumento da concentração do surfactante. No entanto, a razão da intensidade das bandas III/I apresenta um decréscimo de valor quando comparado à emissão em água. Tal observação é atribuída à incorporação do antraceno num microambiente de menor polaridade, em função da formação de pré-agregados e micelas, do surfactante em soluções

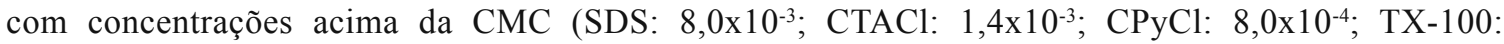
$2,6 \times 10^{-4} \mathrm{~mol} \mathrm{~L}^{-1}$ ). Os espectros de emissão não mostram o aparecimento de excímeros (emissão em $\lambda_{\text {antraceno }}=560 \mathrm{~nm}$ ) e deslocamento espectral das bandas de (I) a (IV). Na presença de CPyCl a banda (I) é totalmente suprimida e as bandas (II), (III) e (IV) decrescem de intensidade com o aumento da concentração do surfactante. Este comportamento sugere que o antraceno se encontra na região da interface da micela-água associado ao anel aromático do surfactante. 

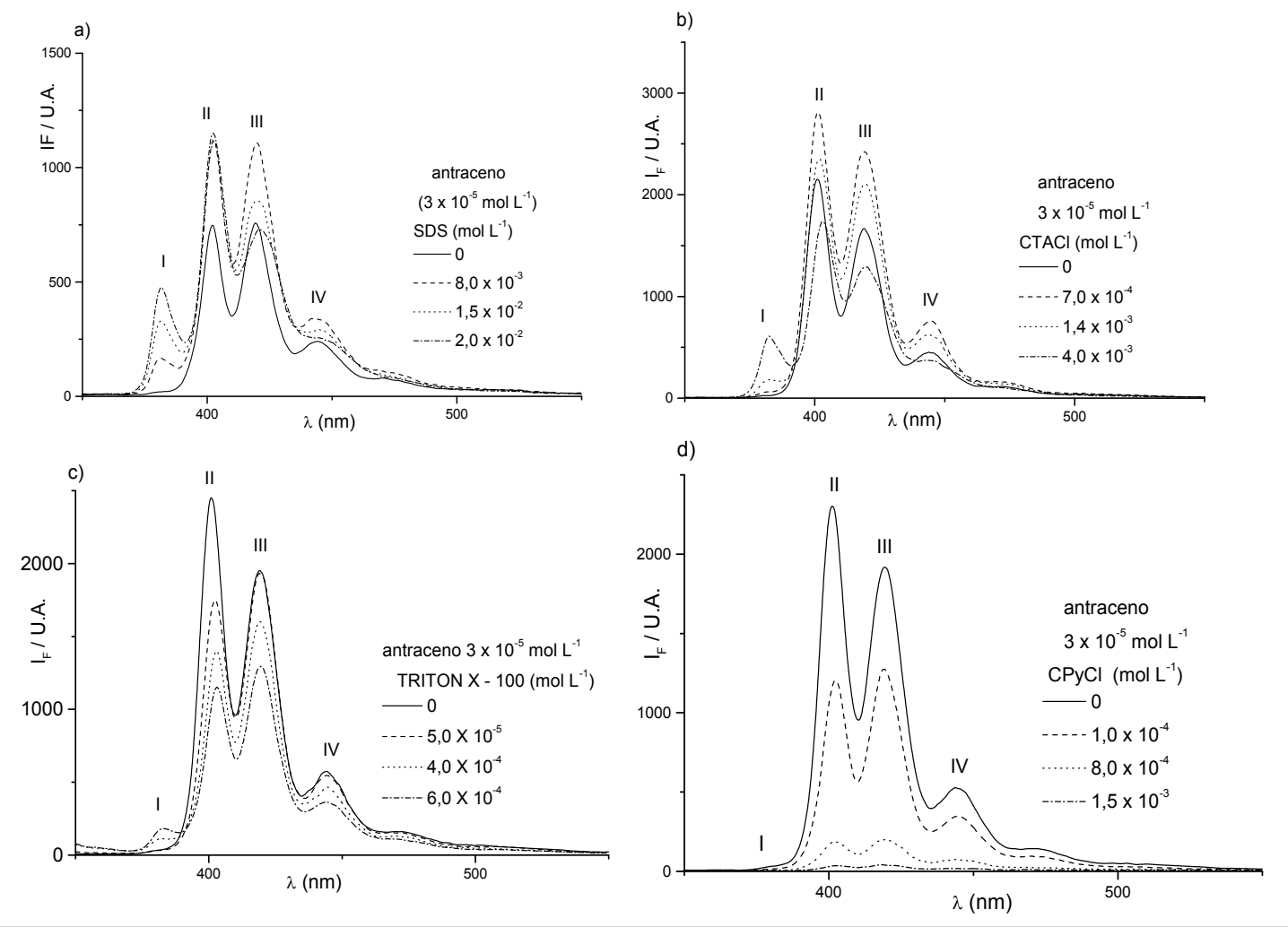

Figura 2. Espectro de fluorescência do antraceno em solução com adição de surfactante:a) SDS; b) CTACl; c) TX-100; d) $\mathrm{CPyCl}, \lambda_{\text {exc }}=260 \mathrm{~nm}$.

Na Figura 3a-d estão representadas as intensidades de fluorescência das bandas (I) e (IV) em função da concentração de cada surfactante. As bandas (I) e (IV) foram empregadas para esta análise pelo fato das variações na sua intensidade de emissão refletirem o processo de agregação/desagregação do antraceno. A banda (IV) foi empregada pelo fato de sua emissão estar localizada na região de excímeros estáticos $\left(\lambda_{\text {antraceno }}=445 \mathrm{~nm}\right)$. A banda (I) apresenta comportamento similar para os sistemas antraceno-SDS, antraceno-CTACl e antraceno-TX-100. No entanto, esta banda mostra um aumento significativo de intensidade de fluorescência para concentrações superiores a CMC (Figura 3 a-c). Por outro lado, para a banda (IV) (Figura 3 a-d) verifica-se a diminuição de intensidade de fluorescência com o aumento da concentração do surfactante. Este comportamento permite sugerir a presença de restrições às transições eletrônicas de menor energia, causadas por distorções impostas às moléculas do antraceno incorporadas aos microdomínios apolares das micelas. 

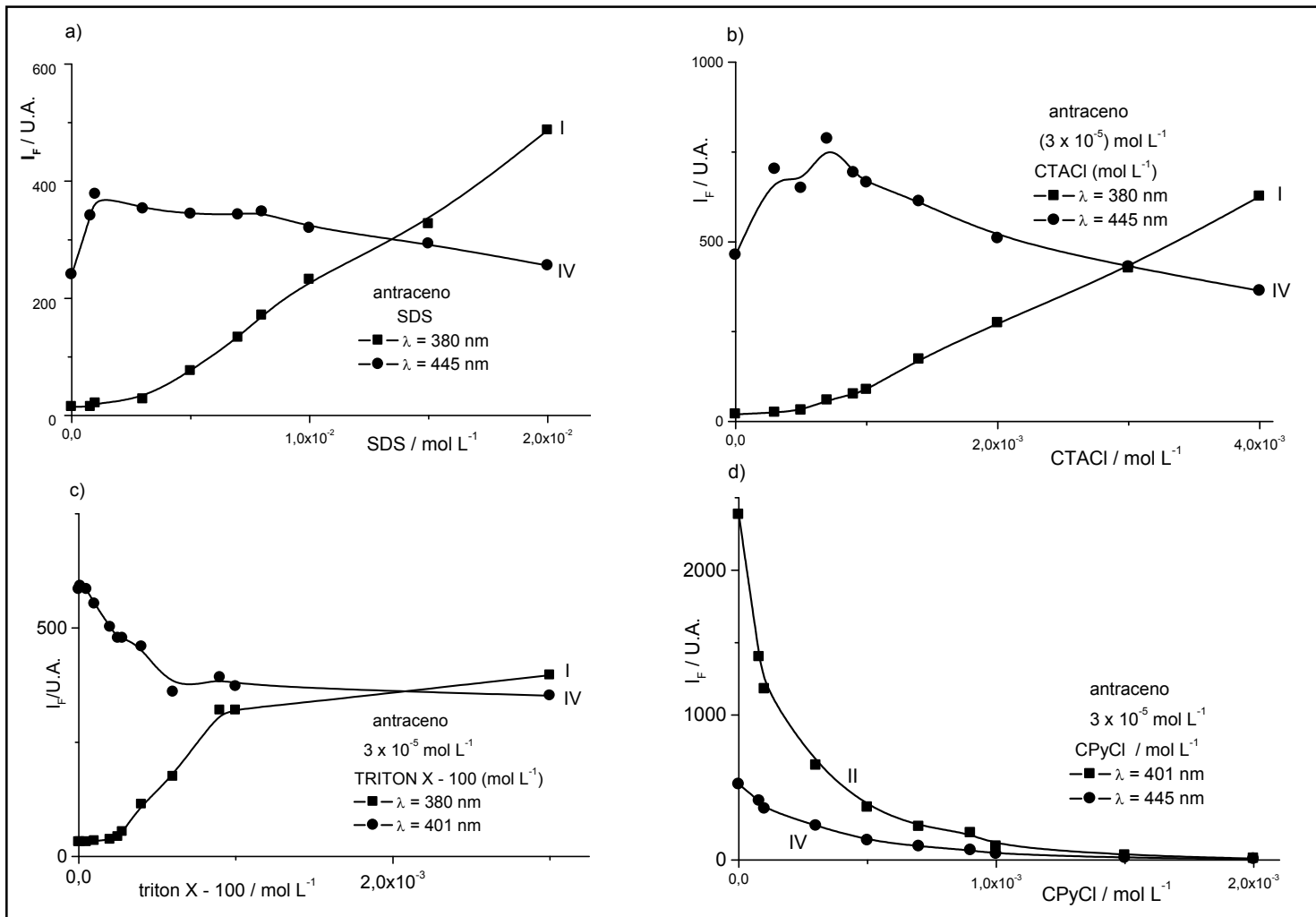

Figure 3. Intensidade de fluorescência do antraceno em função do aumento da concentração de surfactante: a) SDS; b) CTACl; c) TX-100; d) CPyCl.

\section{Comportamento do sistema-antraceno-argila}

A Figura 4 mostra os espectros de emissão de fluorescência do antraceno em suspensão aquosa de argila. Os espectros de emissão em função do aumento da concentração do antraceno não apresentam mudança estrutural. Para o sistema antraceno-argila se observa: a) a ausência da emissão da banda (I) em $380 \mathrm{~nm}$; b) a diminuição expressiva da intensidade de emissão de fluorescência das bandas (II - IV) em relação ao sistema antraceno-água. Estes efeitos podem ser atribuídos à: a) supressão da emissão de fluorescência causada pela argila, e b) a reabsorção da emissão do antraceno presente nos microdomínios apolares na superfície da argila, mesmo para baixas concentrações, induzindo a formação de agregados estáticos não fluorescentes do antraceno. 


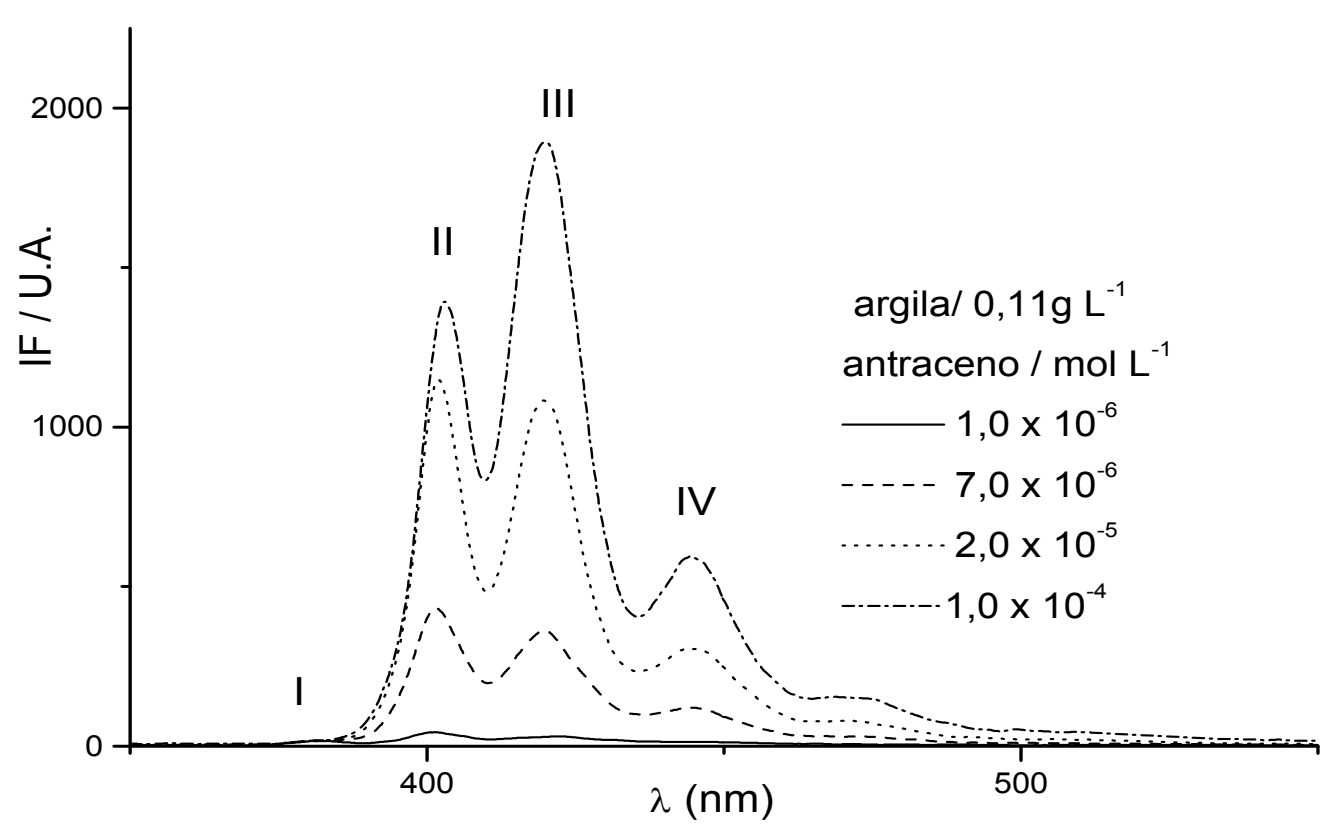

Figura 4. Espectro de fluorescência do antraceno em suspensão aquosa de argila, $\lambda_{\text {exc }}=260 \mathrm{~nm}$. 


\section{Comportamento do sistema antraceno-argila-surfactantes}

Os espectros de fluorescência do antraceno em suspensão de argila com adição dos surfactantes SDS, CTACl, CPyCl e TRITON X-100 estão apresentados na Figura 5 a-d.

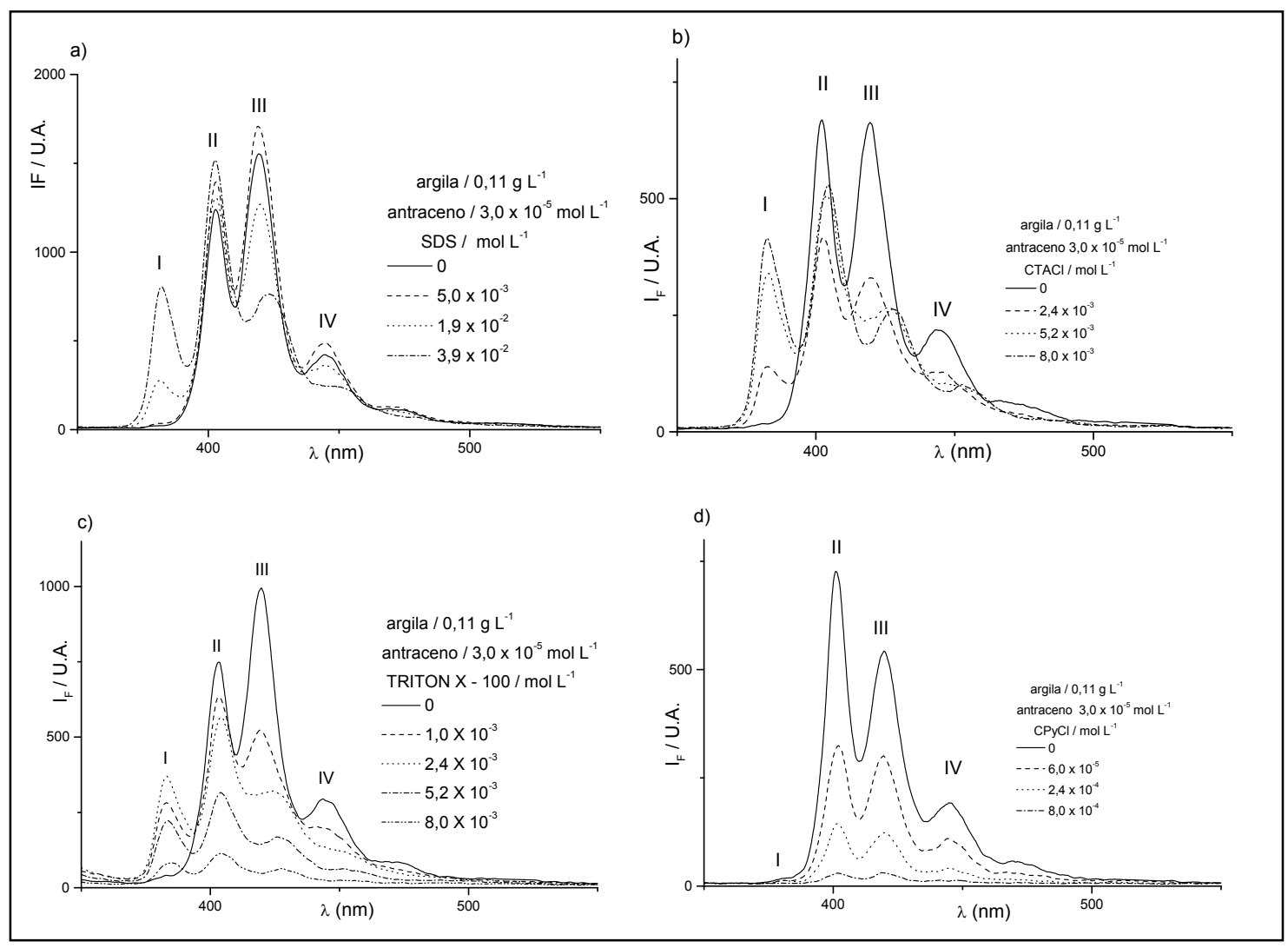

Figura 5. Espectro de fluorescência do sistema antraceno-argila-surfactante com o aumento da concentração do surfactante: a) SDS; b) CTACl; c) TX-100; d) CPyCl, $\lambda_{\text {exc }}=260 \mathrm{~nm}$.

A mudança de meio onde se localiza a sonda afeta o espectro de emissão, o que pode ser verificado para o antraceno a partir da mudança da estrutura fina de emissão de fluorescência mostrada pelo aumento da concentração dos surfactantes. Para o sistema antraceno-argila em presença dos surfactantes, SDS, CTACl e TRITON X - 100 (Figura 5 a-c), observa-se a intensificação da banda (I) em $380 \mathrm{~nm}$. A intensificação da banda (I) é atribuída à incorporação do antraceno nos microdomínios hidrofóbicos das micelas dos surfactantes, diminuindo o efeito de reabsorção; simultaneamente, se observa um decréscimo das bandas (III) e (IV) (Figura 6 a-c). 

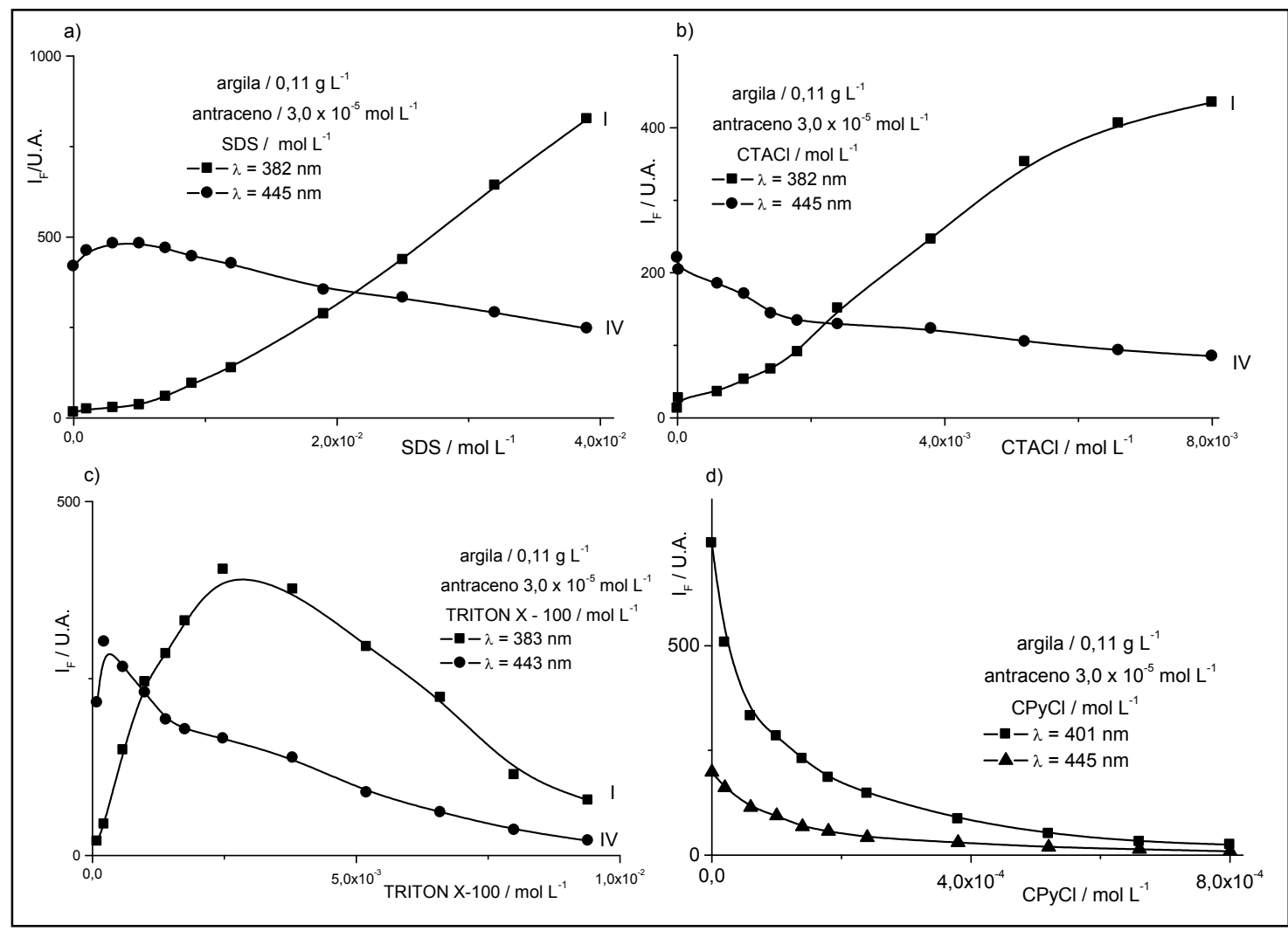

Figure 6. Intensidade de fluorescência do antraceno, em suspensão de argila, em função do aumento da concentração de surfactante: a) SDS; b) CTACl; c) TX-100; d) CPyCl.

A intensidade de fluorescência de todas as bandas vibracionais do antraceno em $\mathrm{CPyCl}$ (Figura 6 d) diminui com o aumento da concentração do surfactante tendo sido este efeito atribuído ao efeito de supressão causado pela presença do CPyCl. Para os sistemas estudados empregando os surfactantes SDS, CTACl e Triton X-100, as intensificações da banda (I) observadas nos espectros de emissão do antraceno estão relacionadas com a concentração micelar crítica dos surfactantes.

Para o sistema antraceno-argila-surfactante, empregando o TX-100, verifica-se um ponto de máximo de emissão de fluorescência para a banda (I), para a concentração de $3,0 \times 10^{-3} \mathrm{~mol} \mathrm{~L}^{-1}$, seguido da diminuição de sua intensidade. Este fenômeno de diminuição da fluorescência da banda (I) pode estar associado ao processo de reabsorção de energia radiante pela molécula do surfactante.
Supressão da emissão de fluorescência do antraceno nos sistemas: antraceno-água, antracenoargila, antraceno-argila-surfactante na presença de íons metálicos (M(II))

A Figura 7 a-b apresenta os o comportamento da banda (II), para os sistemas antraceno-água-M(II) e antraceno-argila-M(II), M(II)= $\mathrm{Cu}(\mathrm{II}) ; \mathrm{Cd}(\mathrm{II}) ; \mathrm{Hg}(\mathrm{II}) ; \mathrm{Ni}(\mathrm{II}) ; \mathrm{Pb}$ (II), como função do aumento da concentração dos íons $(0.0166 \leq$ $\left.\mathrm{C}_{\mathrm{M}(\mathrm{II})} \leq 1.92 \times 10^{-2}\right) \mathrm{mol} \mathrm{L}^{-1}$. Uma avaliação da Figura 7 a permite observar que todos os íons M(II), de modo geral, promovem a supressão da fluorescência do antraceno. Para baixas concentrações dos íons M(II) verifica-se de partida uma considerável supressão da intensidade da fluorescência da banda (II). Com o aumento da concentração, $\mathrm{C}_{\mathrm{M}(\mathrm{II})}>5,0 \times 10^{-3} \mathrm{~mol} \mathrm{~L}^{-1}$, o valor da intensidade de 
fluorescência permanece aproximadamente constante.

A eficiência na supressão da fluorescência da banda (II) obedece as seguintes ordens: 1- para baixas concentrações $\left(\left(0.0166 \leq \mathrm{C}_{\mathrm{M}(\mathrm{II})} \leq 1.2 \times 10^{-3}\right)\right.$ mol L $\left.{ }^{-1}\right): \mathrm{Cu}(\mathrm{II})>\mathrm{Pb}(\mathrm{II})>\mathrm{Hg}(\mathrm{II})=\mathrm{Ni}(\mathrm{II})>\mathrm{Cd}(\mathrm{II})$; 2- para concentrações $>1,2 \times 10^{-3} \mathrm{~mol} \mathrm{~L}^{-1}: \mathrm{Cu}(\mathrm{II})>$ $\mathrm{Hg}(\mathrm{II})>\mathrm{Ni}(\mathrm{II})>\mathrm{Pb}(\mathrm{II})=\mathrm{Cd}(\mathrm{II})$. Os resultados de supressão permitem sugerir que os íons de $\mathrm{Hg}$ (II) e $\mathrm{Cu}(\mathrm{II})$ apresentam-se como melhores agentes para a supressão da fluorescência. Tal fato é corroborado pelo elevado potencial de redução destes íons e o baixo valor de $\mathrm{pKa}$, em comparação aos demais.

Para os sistemas contendo argila em suspensão, Figura $7 \mathbf{b}$, sistema antraceno-argilaM(II), verifica-se comportamento semelhante ao observado para a Figura 7 a, porém, a supressão da fluorescência da banda (II) ocorre com maior intensidade. A fluorescência é reduzida de $90 \%$ para a adição do íon $\mathrm{Pb}(\mathrm{II}), \mathrm{C}_{\mathrm{Pb}(\mathrm{II})} 1,0 \times 10^{-4} \mathrm{~mol}$ $\mathrm{L}^{-1}$, permanecendo aproximadamente constante para as concentração superiores. Para os demais íons metálicos, na mesma concentração, observase uma atenuação em torno de $50 \%$ para o íon $\mathrm{Cu}(\mathrm{II})$ e de aproximadamente $25 \%$ para os íons
$\mathrm{Cd}(\mathrm{II}), \mathrm{Hg}(\mathrm{II})$ e Ni(II). Para valores de concentração superiores a $1,0 \times 10^{-4} \mathrm{~mol} \mathrm{~L}^{-1}$, de modo geral, as intensidades de fluorescência continuam diminuindo assintoticamente.

Uma comparação dos resultados descritos para o sistema antraceno-argila-M(II) com aqueles do sistema antraceno-água-M(II) permite evidenciar uma alteração na ordem de eficiência da supressão da banda (II), conforme segue: $\mathrm{Pb}(\mathrm{II})>$ $\mathrm{Cu}(\mathrm{II})=\mathrm{Hg}(\mathrm{II})>\mathrm{Ni}(\mathrm{II})=\mathrm{Cd}(\mathrm{II})$.

A modificação na ordem de eficiência na supressão da fluorescência da banda (II) está, provavelmente, relacionada ao efeito hidrofóbico que favorece a interação antraceno-argila. A presença do antraceno e dos cátions metálicos na superfície da argila, com os cátions ocupando os sítios de troca, favorecem a maior aproximação das espécies químicas e a conseqüente supressão da fluorescência da banda (II) do antraceno. O esquema abaixo mostra o equilíbrio entre as espécies químicas na superfície da argila, para o sistema antraceno-argila-M(II):
1) $\mathrm{L}+\operatorname{argila}^{\mathrm{n}-} \leftrightarrows \mathrm{L}---\operatorname{argila}^{\mathrm{n}-}$;2) $\mathbf{M}^{\mathrm{n}+}+\operatorname{argila}^{\mathrm{n}-} \leftrightarrows$
M---argila; 3) $\mathbf{L}+\mathbf{M}^{\mathrm{n}+}+\operatorname{argila}^{\mathrm{n}-} \leftrightarrows \quad \mathrm{L}-\mathrm{M}-$
argila

$\mathrm{L}=$ antraceno; $\mathrm{M}=$ íon metálico.

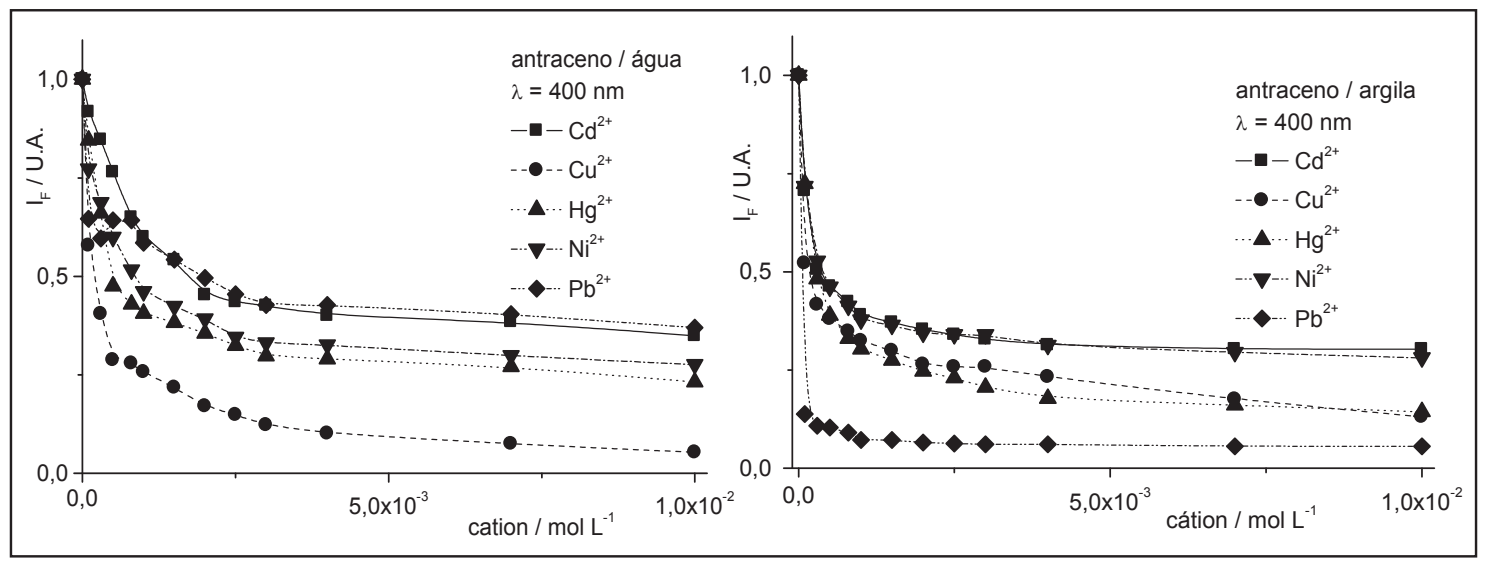

Figura 7: a) Intensidade de fluorescência do antraceno, em função do aumento da concentração dos cátions metálicos; 7b) Intensidade de fluorescência do antraceno, em suspensão de argila, em função do aumento da concentração dos cátions metálicos, $\lambda_{\mathrm{ex}}=260 \mathrm{~nm}$.

Nos sistemas formados por argila em suspensão e surfactantes, Figuras 8a e 8b, semelhante à Figura 7 a-b, apresenta a intensidade de fluorescência do antraceno no sistema antraceno-argila-surfac- 
tante, empregando os surfactantes SDS (aniônico) e o CTACl (catiônico), como sistemas representativos dos demais surfactantes. Observa-se a partir da figura que a intensidade de fluorescência da banda (II) diminui com o aumento da concentração dos cátions metálicos. A supressão da fluorescência encontra sua maior efetividade para o sistema antraceno-argila-SDS, na presença dos íons $\mathrm{Pb}$ (II) e $\mathrm{Cu}$ (II) (Figura 8 a) e para o $\mathrm{Hg}$ (II) no sistema antraceno-argila-CTACl (Figura 8 b). O SDS, aniônico, apresenta fraca interação com a superfície da argila e o antraceno pode estar localizado prioritariamente nos microdomínios hidrofóbicos formados pelos agregados de surfactantes no seio da solução. Assim, o antraceno estando protegido nos microdomínios hidrofóbicos o efeito supressor é similar àquele observado nas soluções aquosas diluídas. Desta forma, os íons de
$\mathrm{Pb}(\mathrm{II})$ e $\mathrm{Cu}(\mathrm{II})$ apresentam-se como os melhores supressores em SDS pela maior associação com as cargas negativas do grupo polar da micela.

O CTACl, surfactante catiônico, apresenta forte interação com a superfície da argila sendo que o antraceno pode estar localizado nos microdomínios hidrofóbicos, gerados pelo surfactante na superfície da argila, ou localizado nos agregados do surfactante no seio da solução. Assim, a adição dos cátions metálicos exerce menor efeito na supressão da emissão do antraceno em relação ao observado no sistema antraceno-argila-SDS. O mercúrio, ao contrário dos demais cátions metálicos, promove a floculação do sistema antracenoargila-CTACl observando-se acentuado decréscimo da emissão nas primeiras adições do cátion metálico. O sistema floculado era visível a olho nu.

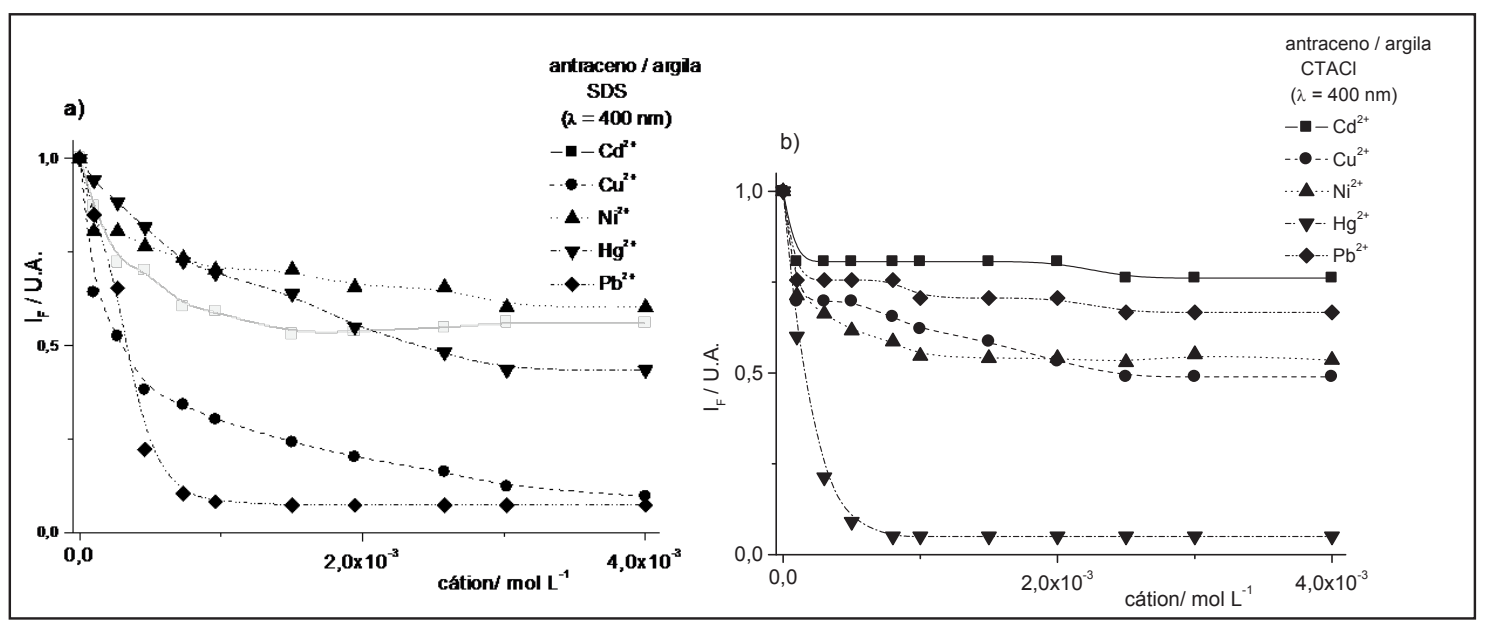

Figura 8. Intensidade de fluorescência do antraceno, em suspensão de argila, em função do aumento da concentração dos cátions metálicos, na presença dos surfactantes: a) SDS, b) CTACl

\section{Conclusões}

Os parâmetros de emissão avaliados fornecem subsídios para o entendimento da adsorção de surfactantes e cátions metálicos na superfície hidrofílica de argilas. Contribuindo, dessa forma, para um melhor entendimento da interação surfactantes-argila em suspensão aquosa, uma vez que as argilas modificadas organicamente apresentam-se eficientes na solubilização de compostos orgâni- cos apolares. O caráter hidrofóbico da sonda define, portanto, a eficiência de sua interação com os microdomínios criados pelos surfactantes ou com a superfície da argila.

Os resultados obtidos para os ensaios de supressão permitem sugerir que a superfície siloxano da argila possui sítios de adsorção que complexam ácidos mais duros, p.e., o íon $\mathrm{Cu}(\mathrm{II})$. O íon uma vez fixado ao substrato da argila não é facilmente trocado pelas espécies orgânicas (catiônicas/aniônicas/não iônica) presentes no meio 
reacional. As espécies orgânicas podem, preferencialmente, coordenarem-se ao íon metálico, sendo que a intensidade desta interação é dependente da habilidade do soluto orgânico em competir com outros sítios de coordenação; sítios disponíveis na superfície da argila próximos ao íon metálico.

Nos ensaios de supressão da fluorescência da banda (II) no sistema antraceno-água-M(II) o íon $\mathrm{Cu}(\mathrm{II})$ apresentou-se como o supressor mais eficiente. Os íons $\mathrm{Cd}(\mathrm{II})$ e $\mathrm{Ni}$ (II) apresentaramse como os de menor eficiência. A intensidade da supressão causada pelos íons $\mathrm{Cu}$ (II) é devida a contribuição de supressão estática e dinâmica do processo fotoquímico. Nos sistemas antraceno-
argila-surfactante-M(II), surfactante catiônico, os íons de $\mathrm{Hg}$ (II) promoveram a máxima supressão da fluorescência da banda (II) e a concomitante floculação do sistema. Situação semelhante foi observada para o sistema preparado empregandose surfactante aniônico na presença de íons $\mathrm{Pb}(\mathrm{II})$. Tal comportamento permite sugerir a aplicação de sistemas desta natureza para a remoção de hidrocarbonetos aromáticos policíclicos de água e águas residuais.

R. Magri, F.L. Fertonani, I.A. Pastre. Photophysical behavior of anthracene in micro-heterogeneous systems clay-surfactant-metallic ions

\begin{abstract}
Clays are a class of complex micro-heterogeneous and can be used as a substrate for adsorption. The sorption behavior of solid-phase enhanced by the presence of surfactants, organoclay, is an important phenomenon studied by environmental technology for removal of polycyclic aromatic hydrocarbons (PAHs) anthropogenic sources from water. This work aims to study the photophysical behavior of anthracene as a model of HPA systems in microheterogeneous clay-surfactant-metal ions (M (II) = Cd (II), Cu (II), $\mathrm{Hg}$ (II), Ni (II) and Pb (II); surfactants: CTACl; SDS; TR-X100). The experimental assays were conducted by monitoring changes in the static properties of fluorescence and anthracene emission suppression; anthracene was used as fluorescent probe. $\mathrm{M}$ (II)ions, like $\mathrm{Cd}(\mathrm{II}), \mathrm{Cu}(\mathrm{II}), \mathrm{Hg}$ (II), $\mathrm{Ni}$ (II) and $\mathrm{Pb}$ (II)were used as anthracene fluorescence suppressors. The profile of the fluorescence spectrum and the results of the suppression of fluorescence of the probe allowed inferring the specific place of the anthracene solubilization into the micro-heterogeneous systems studied and the consequent arrangement of them.
\end{abstract}

Keywords: anthracene; clay; suppression; fluorescence; metallic ions.

\section{Referências}

[1 ] D. AVNIR; R. BUSSE, M. OTTOLENGH, E. WELLNER, K. A. ZACHARIASSE, Fluorescent probes for silica and reversed-phase silica surfaces: 1,3-di-1-pyrenylpropane and pyrene. Journal of Physical Chemistry. 89 (1985) 35213526.

[2 ] P. DE MAYO, L. V. NATARAJAN, W. R. WARE, Surface photochemistry: the effects of temperature on the singlet quenching of pyrene adsorbed on silica gel by 2-bromonaphthalene. Journal of Physical Chemistry. 89 (1985) 35263530.

[3] R. A. DELLA GUARDIA, J. K. THOMAS, Photoprocesses on colloidal clay systems: micelles with colloidal montmorillonite, Journal of Physical Chemistry, 88 (1984) 964970.

[4] D. J. VAUGHAN, R. A. D. PATTRICK, Mineral surfaces. London: Chapman \& Hall, 9 (1995) 303-332.
[5] F. WILKINSON, D. R.WORRALL, S. L. WILLIAMS, Primary photochemical processes of anthracene adsorbed on silica gel. Journal of Physical Chemistry, 99 (1995) 66896696.

[6 ] R. A. SCHOONHEYDT, T. PINNAVAIA, G. LAGALY, N. GANGAS, Pillared clays and pillared layered solids. Pure and Applied Chemistry, 71(12) (1999) 2367-2371.

[7] T. HAYASHI, N. MATAGA, I. SAKATA, S. MISUMI, M. MORITA, J. TANAKA, Excimer fluorescence and photodimerization of anthracenophanes and 1,2-dianthrylethanes. Journal of the American Chemical Society, 98 (1976) 5910.

[8] I. A. PASTRE, G. R. CUCOLO, Efeitos do meio nos processos fotoquímicos e fotofísicos dos derivados catiônicos do antraceno. Revista de Iniciação Científica: XI Congresso de iniciação científica da UNESP, 1 (2000) 117-124.

[9] H. BOUAS-LAURENT, A. CASTELlan, J. P. DESVERGNE, From anthracene photodimerization to jaw photochromic materials and photocrowns. Pure and Applied Chemistry, 52 (1980) 2633-2648. 
[10] A. CORTI, P. CINELLI, S. D'ANTONE, E. R. KENAVY, R. SOLANO, Biodegradation of poly(vinyl alcohol in soil environment: influence of natural organic fillers and structural parameters. Macromolecular Chemistry and Physics, 203 (2002 ) 1526-1531.

[11] F. GESSNER, C. C. SHIMITT, Estudos das interações entre corantes catiônicos em suspensão de várias argilas. 1995. 104 f. Tese (Doutorado em Química) - Instituto de Química, Universidade de São Paulo, São Carlos, 1995.

[12] K. KALYANASUNDARAM, Photochemistry in microheterogeneous systems. Lausanne: Institute of Technology, (1987) 338.

[13] J. E. HUHEEY, Inorganic chemistry: principles of structure and reativity. London, Harper \& Row, (1975) 737.

[14] PASTRE, I.A.; NASCIMENTO OLIVEIRA, I.; MOITINHO, A.B.S.; SOUZA, G.R.; IONASHIRO, E.Y.; FERTO-

NANI, F.L. "Thermal behaviour of intercalated 8-hydroxyquinoline (oxine) in montmorillonite clay. Journal of Thermal Analysis and Calorimetry, 75 (2004) 663-670.

[15] NEWMANN, M.G.; GUESNER, F.; CIONE,A.P.P.;SARTORI, R.A.; SHIMITT CAVALHEIRO, C.C. "Interação entre corantes e argilas em suspensão aquosa". Química Nova, v. 23 (2008) 818-830. 